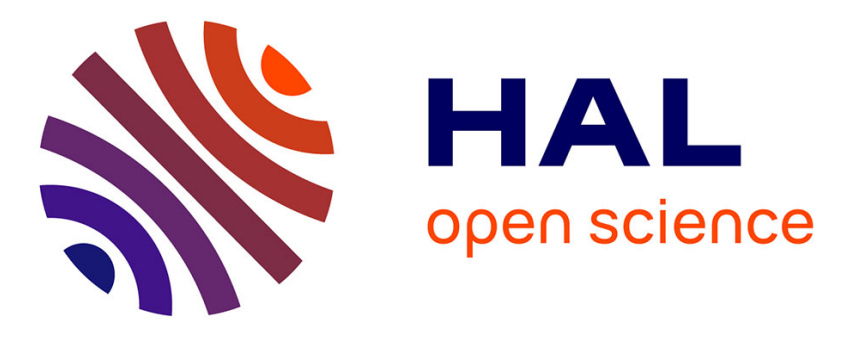

\title{
4D deconvolution and demixing for supernova follow-up
}

\author{
Sébastien Bongard, Éric Thiébaut, Ferréol Soulez, Emmanuel Pecontal
}

\section{To cite this version:}

Sébastien Bongard, Éric Thiébaut, Ferréol Soulez, Emmanuel Pecontal. 4D deconvolution and demixing for supernova follow-up. the First IEEE GRSS Workshop on Hyperspectral Image and Signal Processing - Evolution in Remote Sensing - (WHISPERS'09), Aug 2009, Grenoble, France. pp.0-0. hal-00436646v2

\section{HAL Id: hal-00436646 \\ https://hal.science/hal-00436646v2}

Submitted on 4 Jan 2010

HAL is a multi-disciplinary open access archive for the deposit and dissemination of scientific research documents, whether they are published or not. The documents may come from teaching and research institutions in France or abroad, or from public or private research centers.
L'archive ouverte pluridisciplinaire HAL, est destinée au dépôt et à la diffusion de documents scientifiques de niveau recherche, publiés ou non, émanant des établissements d'enseignement et de recherche français ou étrangers, des laboratoires publics ou privés. 


\section{D DECONVOLUTION AND DEMIXING FOR SUPERNOVA FOLLOW-UP}

\author{
S. Bongard \\ LPNHE - place Jussieu \\ 75252 Paris Cedex 05 - France
}

\author{
E. Thiébaut, F. Soulez, E.Pécontal
}

\begin{abstract}
We present an inverse problem approach to jointly solve a problem of deconvolution and demixing of sources from 4D $(x, y, \lambda, t)$ astronomical data obtained by observing a supernova and its host galaxy at different epochs. In order to obtain supernova spectra of high photometric quality, we take special care of avoiding demixing biases and deconvolution artifacts caused by the very limited size of the field of view. We assert the performances of our method on realistic simulated data.
\end{abstract}

Index Terms - supernova survey; inverse problem; demixing; deconvolution.

\section{INTRODUCTION}

We address the joint problems of the deconvolution of a 4D dataset (2 spatial dimensions + wavelength + time) and demixing of a time varying point source object (a supernova), from a time invariant but spatially structured environment (the host galaxy) and a time varying spatially uniform sky background. These observations are meant to obtain photometrically calibrated spectra needed to improve the use of type Ia supernovæ (SNe Ia) as cosmological probes [1, 2]. The problem of demixing spectra from different sources has been previously addressed in astronomy for 2D spectroscopy obtained with long-slit spectrographs [3,4]. The data we consider here is a multi-wavelength set of multi-epoch images, i.e. a $4 \mathrm{D}$ dataset $(x, y, \lambda, t)$. The real data will be obtained with SNIFS (SuperNova Integral Field Spectrograph) [5], an instrument which has been optimized for automated observation of point sources on a diffuse background over the full optical range at moderate spectral resolution. SNIFS main component is a high-throughput wide-band pure-lenslet integral field unit (IFU) à la TIGER [6]. Each observed supernova yields $N_{t}$ datacubes, including one final reference observed when the supernova has faded away. The spatial coordinates correspond to the positions of the microlenses in the microlens array (MLA), and thus to a specific position in the sky for a given exposure. To each microlens corresponds a spectrum of $N_{\lambda}$ flux values. The point spread function (PSF) is estimated from the simultaneous observation of several field stars which are also used as photometric references to calibrate the measured supernova flux to reference standard
Centre de Recherche Astrophysique de Lyon

CNRS-UMR 5574 - Université Lyon 1 - France stars. In all the following work, we will consider the PSF's known and the fluxes calibrated. Also, due to the small size of the field of view $\left(6^{\prime \prime} \times 6^{\prime \prime}\right)$, the PSF's can be considered as shift invariant.

\section{INVERSE PROBLEM APPROACH}

\subsection{Direct Model}

The 4D model of the observed data is a mixture of three components, the host galaxy, the supernova and the sky background, convolved by the PSF of the instrument:

$$
\begin{aligned}
m(\boldsymbol{a}, \lambda, t)=\iint & {\left[I_{\text {gal }}\left(\boldsymbol{a}^{\prime}, \lambda\right)+F_{\text {sn }}(\lambda, t) \delta\left(\boldsymbol{a}^{\prime}\right)+I_{\text {sky }}(\lambda, t)\right] } \\
& \times h\left(\boldsymbol{a}-\boldsymbol{a}_{\lambda, t}-\boldsymbol{a}^{\prime}, \lambda, t\right) \mathrm{d} \boldsymbol{a}^{\prime}
\end{aligned}
$$

where $\boldsymbol{a}=\left(a_{1}, a_{2}\right)$ is the $2 \mathrm{D}$ view angle, $I_{\text {gal }}(\boldsymbol{a}, \lambda)$ and $I_{\text {sky }}(\lambda, t)$ are the specific intensities of the galaxy and the sky, and $F_{\mathrm{sn}}(\lambda, t)$ is the specific flux of the supernova. The PSF, which norm accounts for absolute and chromatic calibration, is denoted $h(\boldsymbol{a}, \lambda, t)$. The pointing offset of exposure $t$ is $\boldsymbol{a}_{\lambda, t}$, it also accounts for the atmospheric differential refraction (ADR) [7] via its wavelength dependence. In the model prior to convolution, the supernova is a point source and it's position is used as the reference for all other positions in the model.

\subsection{Likelihood and regularization}

Our objective is the recovering of $I_{\text {gal }}, F_{\text {sn }}$ and $I_{\text {sky }}$ given the data and the PSF's. To that end, we adopt a very general inverse problem approach, taking special cares to avoid biases in the extraction of the spectral energy distributions (SED's) of the supernovæ which are meant to be used as cosmological probes. Following standard inverse problem approach [8], the solution is obtained by optimizing a joint criterion:

$$
\boldsymbol{x}^{+}=\underset{\boldsymbol{x}}{\arg \min }\left\{f_{\text {data }}(\boldsymbol{x})+f_{\text {prior }}(\boldsymbol{x})\right\},
$$

where $\boldsymbol{x}$ is the set of all unknowns (galaxy, supernova and sky fluxes), $f_{\text {data }}(\boldsymbol{x})$ is a likelihood term which enforces agreement with the data while $f_{\text {prior }}(\boldsymbol{x})$ is a regularization term which enforces agreement with the priors. 
After discretization and assuming uncorrelated Gaussian noise, the likelihood term reads:

$$
f_{\text {data }}(\boldsymbol{x})=\sum_{k, \ell, t} w_{k, \ell, t}\left[d_{k, \ell, t}-m_{k, \ell, t}(\boldsymbol{x})\right]^{2},
$$

where $m_{k, \ell, t}(\boldsymbol{x})$ is the discretized model, $d_{k, \ell, t}$ is a datum, indices $k, \ell$ and $t$ are for the pixel (2D position), spectral channel and epoch respectively and the $w^{\prime} s$ are statistical weights equal to the reciprocal of the variance of the data.

Owing to the time separation between exposures (each exposure is generally taken during a different night), there is no temporal correlation between the parameters (to the notable exception of the galaxy brightness distribution which is assumed to be constant over time). Since there is no time continuity for our data, we indistinctly use the letter $t$ to denote the time or the exposure index.

The purpose of the regularization term is to supplement missing information (our problem having more unknowns than measurements is ill-posed) and to avoid noise amplification (the deconvolution involved makes our problem ill-conditioned) [9]. Most effective regularization consists in accounting for the continuity of the sought distributions along their dimensions. In our case, we do not enforce any spectral continuity on the point source to avoid biases. We also found that spectral continuity was not necessary for the sky. Besides, the galaxy time invariance and the sky flatness are enforced explicitly. Hence, we only use the regularization term to enforce the spatial and spectral continuity of the galaxy. The spatial continuity of the galaxy is required to cope with the ill-posedness of the deconvolution and because we choose to have a model that covers an area twice as large as the field of view to avoid edge artifacts as the PSF's are of size similar to the field of view. We do not want to favor any spatial anisotropy nor any correlation between spatial and spectral features, hence we use a separable regularization for the galaxy:

$$
\begin{aligned}
f_{\text {prior }}(\boldsymbol{x}) & =\iiint w_{\text {spatial }}^{\text {prior }}(\boldsymbol{a}, \lambda)\left\|\frac{\partial I_{\text {gal }}(\boldsymbol{a}, \lambda)}{\partial \boldsymbol{a}}\right\|^{2} \mathrm{~d} \boldsymbol{a} \mathrm{d} \lambda \\
& +\iiint w_{\text {spectral }}^{\text {prior }}(\boldsymbol{a}, \lambda)\left[\frac{\partial I_{\text {gal }}(\boldsymbol{a}, \lambda)}{\partial \lambda}\right]^{2} \mathrm{~d} \boldsymbol{a} \mathrm{d} \lambda
\end{aligned}
$$

where $w_{\text {spatial }}^{\text {prior }}$ and $w_{\text {spectral }}^{\text {prior }}$ are regularization weights. In practice, we approximate the partial derivatives by finite differences and the integrals by sums over the pixels and spectral index.

For simple 2D image restoration (e.g. deconvolution), stationary priors are generally assumed. Following this prescription, the regularization weights would depend neither on the position nor on the wavelength which would left us with the problem of tuning only two regularization levels: the weight of the spatial regularization and that of the spectral regularization. Owing to the high dynamical range in astronomical images and to avoid grossly over-regularization of large features
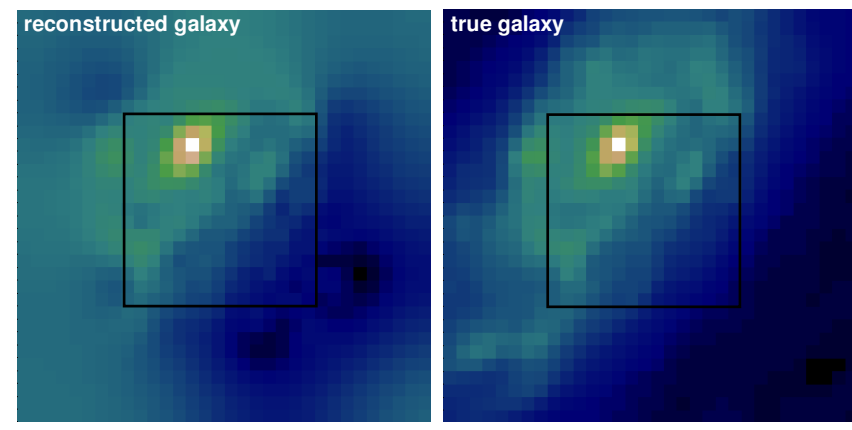

Fig. 1. Reconstructed galaxy vs. true galaxy. Note the very good reconstruction inside of the field of view (shown as a black box) and the field of view extrapolation in the reconstruction.

or under-regularization of small features, we rather suggest that the regularization weights be, at least chromatic. From Bayesian considerations [10], we derive:

$$
\begin{aligned}
w_{\text {spatial }}^{\text {prior }}(\boldsymbol{a}, \lambda) & =\mu_{\text {spatial }}^{\text {prior }} q_{\text {spatial }}^{\text {prior }}(\lambda) \\
w_{\text {spectral }}^{\text {prior }}(\boldsymbol{a}, \lambda) & =\mu_{\text {spectral }}^{\text {prior }} q_{\text {spectral }}^{\text {prior }}(\lambda)
\end{aligned}
$$

with expected values approximated by spatial averages computed for the observed brightness distribution $I_{\mathrm{obs}}(\boldsymbol{a}, \lambda)$ :

$$
\begin{aligned}
& q_{\text {spatial }}^{\text {prior }}(\lambda)=\left[\frac{1}{\Omega} \iint\left\|\frac{\partial I_{\text {obs }}(\boldsymbol{a}, \lambda)}{\partial \boldsymbol{a}}\right\|^{2} \mathrm{~d} \boldsymbol{a}-\text { bias }\right]^{-1} \\
& q_{\text {spectral }}^{\text {prior }}(\lambda)=\left[\frac{1}{\Omega} \iint\left[\frac{\partial I_{\text {obs }}(\boldsymbol{a}, \lambda)}{\partial \lambda}\right]^{2} \mathrm{~d} \boldsymbol{a}-\text { bias }\right]^{-1}
\end{aligned}
$$

where $\Omega$ is the size of the observed field of view. The two bias terms account for the noise propagation and are directly derived from the variance of $I_{\mathrm{obs}}$. The two remaining free parameters $\mu_{\text {spatial }}^{\text {prior }}$ and $\mu_{\text {spectral }}^{\text {prior }}$ are needed because the $q$ 's terms are estimated from the data, not from the true galaxy. We expect that $\mu_{\text {spectral }}^{\text {prior }}$ be of order unity, and that $\mu_{\text {spatial }}^{\text {prior }}$ has to be set to smaller values due to the smoothing by the PSF.

The demixing of the galaxy and the supernova is possible thanks to the spatial regularization of the galaxy (which prevents a sharp feature such as the supernova to be accounted as being part of the galaxy) and to the reference exposure for which the supernova is known to be invisible. However the demixing cannot be perfect and we expect some pollution between the recovered galaxy and supernova. In practice, we found that this bias is not very large. Nevertheless, it may reach levels incompatible with the accuracy required by the scientific goals of SNIFS. In order to better suppress this bias, we first deconvolve the final reference alone with the most adequate set of hyper-parameters and with the supernova explicitly tuned off. Once this reconstruction is achieved, we use it as a prior to fix the galaxy level under the supernova. 


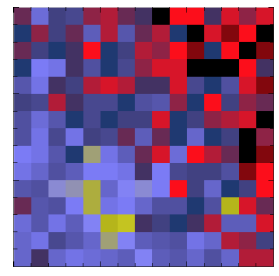

data $(3975 \AA)$

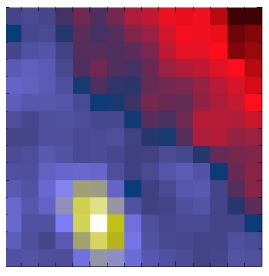

reconstruction

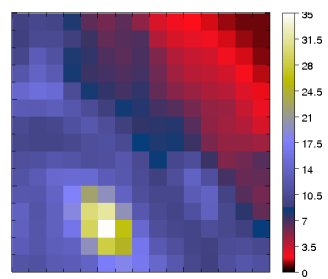

ground truth

Fig. 2. Image of the galaxy in the $3974 \AA$ spectral channel. From left to right: raw data from the final reference, the reconstructed deconvolved galaxy, the true galaxy.
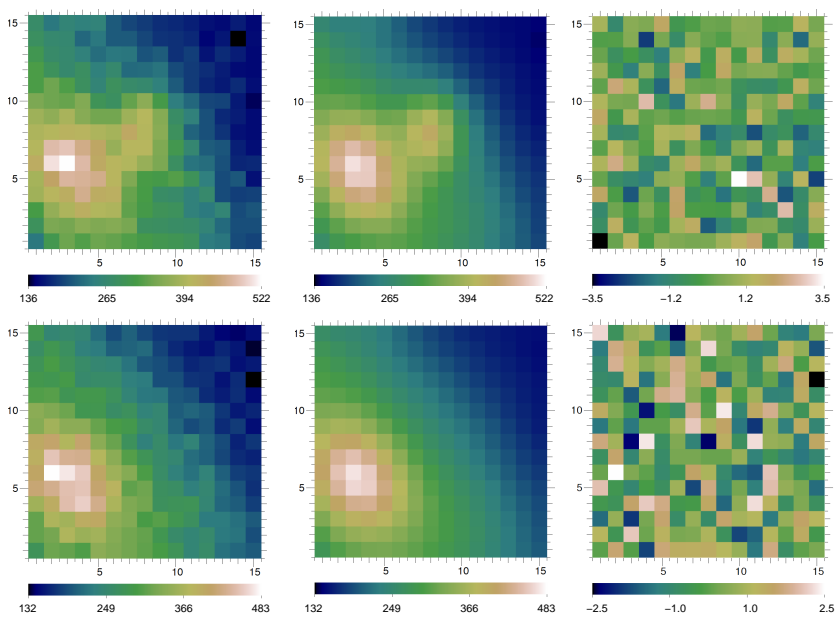

Fig. 3. Results at two different epochs. Top: 10 days before max. Bottom: final reference. From left to right: data, model and residuals.
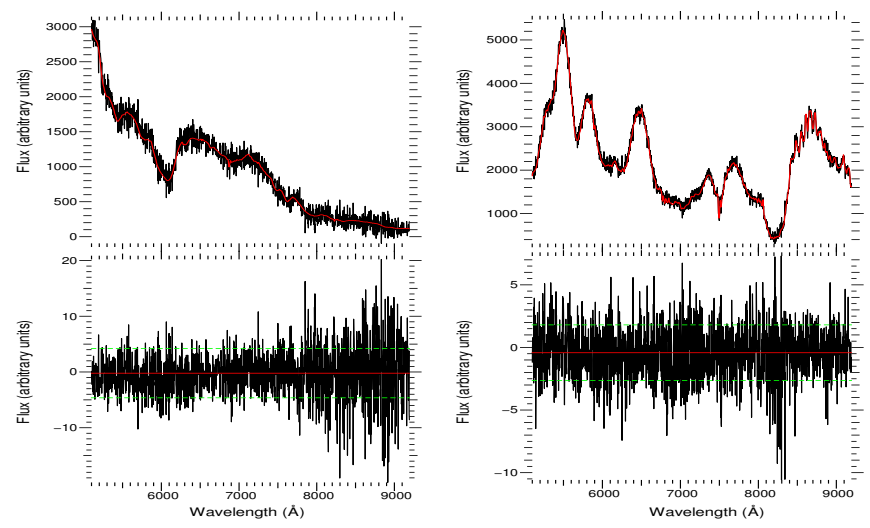

Fig. 4. Top: true (red) and extracted (black) spectra of the super-nova, 10 days before peak brightness (left) and 40 days after peak brightness (right). Bottom: relative difference between the true and extracted spectra.

\section{ALGORITHM AND RESULTS}

\subsection{Algorithm summary}

\subsubsection{Initialization}

The first step is to deconvolve the galaxy from the final reference alone, with the supernova explicitly turned off. The sky and the galaxy specific intensities are defined up to a chromatic offset which can be added to the galaxy and subtracted to the sky with no incidence on the model. In order to lift this degeneracy, the sky in the reference exposure is held fixed to a chosen a priori distribution. This choice is arbitrary, a more realistic sky could be either estimated $a$ posteriori, bearing in mind that it could slightly impact the reconstruction via the spectral regularization.

\subsubsection{Registration}

At this stage, the galactic reconstruction is used to refine the measure of the relative distance between the galaxy and the supernova. This distance is estimated on all exposures via a maximum correlation method between the galaxy model and the data at other epochs but where the supernova has been masked. Using the median of the positions measured, the galaxy is then re-build from the final reference to give $I_{\text {gal }}^{\text {prior }}$ which is used later (see below) as a prior distribution. Since the reference of all positions in the model space is the position of the supernova, this is equivalent to propagating the relative distance between the supernova and the galaxy to all epochs. This step is mandatory because it proved impossible to measure externally the position of the supernova in each exposure with the accuracy needed by the simultaneous treatment of all the exposures.

\subsubsection{Simultaneous demixing of all epochs}

Once these preparation steps have been taken, we globally fit for supernova, sky and galaxy on all exposures with a spatial regularization on $I_{\text {gal }}-I_{\text {gal }}^{\text {prior }}$. This is achieved through a hierarchical optimization, where the supernova and sky are extracted for each change in the galaxy map.

\subsection{Results on simulated data}

\subsubsection{Simulator}

In order to test the results of our algorithm, and especially to assert that the photometric bias introduced by the extraction is negligible, the true supernova spectrum is required. This is only possible through simulated data. The simulator we implemented generates a supernova time series and places it at any given position on top of a realistic galaxy. The spectrum of the galaxy for each one of its pixels is obtained by fitting a stellar population library to real multi-color images. 
This method yields a galaxy model that has a realistic spectrospatial distribution. The supernova and the galaxy are then convolved by a PSF obtained from a library of real PSF's and a sky spectrum is added to the exposure. Photon noise (with Poisson statistic) and read out noise (with Gaussian statistic) are finally added to produce the data.

\subsubsection{Results}

We processed all exposures (here 11 exposures, including one final reference) and all wavelengths (here 780 spectral channels) simultaneously with our algorithm to perform the joint deconvolution and demixing. Figure 1 shows that the reconstruction inside of the field of view captures all the small scale galactic structure without noticeable pollution from the supernova, that would mean a bias in the SN Ia spectra extracted. Also note that the extrapolation outside the field of view is quite consistent with the real distribution. Figure 2 stresses on a single wavelength channel corresponding to an absorption feature (i.e. where the galaxy has a low signal to noise ratio) to show the gain in quality obtained by exploiting the spectral continuity of the galaxy. In Figure 3 we display one wavelength channel of two simulated exposures: an exposure containing the galaxy and the supernova, and an exposure containing only the galaxy. Since no structured features are left in the residuals, our method accounts correctly for all the structures of the object. Figure 4 displays the supernova spectra at different epoch, it shows that the extraction is unbiased and of photometric quality. Moreover, both spectra come from epochs with very different PSF's and ADR's, showing the accuracy of the field extrapolation, needed to subtract correctly the different amount of light brought in the field of view by the wings of the PSF.

\section{CONCLUSION}

We presented an algorithm to deconvolve and separate components from a multi-wavelength multi-epoch 4D dataset. Our method is based on an inverse problem approach, it processes all exposures and all wavelengths simultaneously and exploits the existing spatial, temporal and/or spectral continuities or invariances in the different light sources (galaxy, super nova and sky background) to improve demixing quality and avoid deconvolution artifacts. Our algorithm yields an extraction of the component of interest, i.e. the supernova spectrum, with high photometric accuracy.

\section{Acknowledgments}

This work was supported in part by a grant from the Gordon $\&$ Betty Moore Foundation, and by the Director, Office of Science, Office of High Energy and Nuclear Physics, of the U.S. Department of Energy (DOE) under Contract Nos. DEAC02-05CH11231 and DE-FG02-06ER06-04. We would like to thank D. Munro for freely distributing is Yorick programming language (available at http://yorick.sourceforge.net/).

\section{REFERENCES}

[1] A. G. Riess, A. V. Filippenko, P. Challis, A. Clocchiatti, A. Diercks, P. M. Garnavich, R. L. Gilliland, C. J. Hogan, S. Jha, R. P. Kirshner, B. Leibundgut, M. M. Phillips, D. Reiss, B. P. Schmidt, R. A. Schommer, R. C. Smith, J. Spyromilio, C. Stubbs, N. B. Suntzeff, and J. Tonry, "Observational Evidence from Supernovae for an Accelerating Universe and a Cosmological Constant," AJ, vol. 116, pp. 1009-1038, Sept. 1998.

[2] S. Perlmutter, G. Aldering, G. Goldhaber, R. A. Knop, P. Nugent, P. G. Castro, S. Deustua, S. Fabbro, A. Goobar, D. E. Groom, I. M. Hook, A. G. Kim, M. Y. Kim, J. C. Lee, N. J. Nunes, R. Pain, C. R. Pennypacker, R. Quimby, C. Lidman, R. S. Ellis, M. Irwin, R. G. McMahon, P. Ruiz-Lapuente, N. Walton, B. Schaefer, B. J. Boyle, A. V. Filippenko, T. Matheson, A. S. Fruchter, N. Panagia, H. J. M. Newberg, W. J. Couch, and The Supernova Cosmology Project, "Measurements of Omega and Lambda from 42 High-Redshift Supernovae," APJ, vol. 517, pp. 565-586, June 1999.

[3] F. Courbin, P. Magain, M. Kirkove, and S. Sohy, "A Method for Spatial Deconvolution of Spectra," Astrophysical Journal, vol. 529, pp. 1136-1144, Feb. 2000.

[4] L. B. Lucy and J. R. Walsh, "Iterative Techniques for the Decomposition of Long-Slit Spectra," Astronomical Journal, vol. 125, pp. 2266-2275, Apr. 2003.

[5] G. Aldering, G. Adam, P. Antilogus, P. Astier, R. Bacon, S. Bongard, C. Bonnaud, Y. Copin, D. Hardin, F. Henault, D. A. Howell, J.-P. Lemonnier, J.-M. Levy, S. C. Loken, P. E. Nugent, R. Pain, A. Pecontal, E. Pecontal, S. Perlmutter, R. M. Quimby, K. Schahmaneche, G. Smadja, and W. M. Wood-Vasey, "Overview of the Nearby Supernova Factory," in Society of Photo-Optical Instrumentation Engineers (SPIE) Conference Series, J. A. Tyson and S. Wolff, Eds., Dec. 2002, vol. 4836 of Society of Photo-Optical Instrumentation Engineers (SPIE) Conference Series, pp. 61-72.

[6] R. Bacon, G. Adam, A. Baranne, G. Courtes, D. Dubet, J. P. Dubois, E. Emsellem, P. Ferruit, Y. Georgelin, G. Monnet, E. Pecontal, A. Rousset, and F. Say, "3D spectrography at high spatial resolution. I. Concept and realization of the integral field spectrograph TIGER.," $A \& A$, vol. 113, pp. 347-+, Oct. 1995.

[7] A. V. Filippenko, "The importance of atmospheric differential refraction in spectrophotometry," PASP, vol. 94, pp. 715-721, Aug. 1982. 
[8] D. M. Titterington, "General structure of regularization procedures in image reconstruction," vol. 144, pp. 381$387,1985$.

[9] E. Thiébaut, "Introduction to image reconstruction and inverse problems," in Optics in Astrophysics, R. Foy and F.-C. Foy, Eds., vol. 198 of NATO ASI, pp. 397-422. Kluwer Academic, 2005.

[10] A. Tarantola, Inverse Problem Theory and Methods for Model Parameter Estimation, SIAM, 2005. 